Gut and Liver, Vol. 10, No. 5, September 2016, pp. 665-671

\title{
The Role of Common Pharmaceutical Agents on the Prevention and Treatment of Pancreatic Cancer
}

\author{
Sunil Amin ${ }^{1}$, Paolo Boffetta ${ }^{2}$, and Aimee L. Lucas ${ }^{1}$ \\ ${ }^{1}$ Henry D. Janowitz Division of Gastroenterology and ${ }^{2}$ Tisch Cancer Center, Icahn School of Medicine at Mount Sinai, New York, NY, USA
}

\begin{abstract}
Survival from pancreatic cancer remains poor. Conventional treatment has resulted in only marginal improvements in survival compared with survival in the previous several decades. Thus, considerable interest has emerged regarding the potential use of common pharmaceutical agents as chemopreventative and chemotherapeutic options. Aspirin, metformin, statins, $\beta$-blockers, and bisphosphonates have biologically plausible mechanisms to inhibit pancreatic neoplasia, whereas dipeptidyl-peptidase 4 inhibitors may promote it. Regardless, real-world epidemiological data remain inconclusive. This review examines the hypotheses, evidence, and current state of the literature for each of these medications and their potential roles in the prevention and treatment of pancreatic cancer. (Gut Liver 2016;10:665-671)
\end{abstract}

Key Words: Pancreas; Malignancy; Adenocarcinoma; Epidemiology; Aspirin; Metformin; Statins; Bisphosphonates; Betablockers; Dipeptidyl-peptidase 4 inhibitors

\section{BACKGROUND}

Pancreatic ductal adenocarcinoma is a lethal disease. In 2014, 46,420 cases were diagnosed in the United States, with 39,590 deaths. ${ }^{1}$ Despite advances in surgical management and chemotherapeutic options, the 5-year survival rate has only improved slightly over the last 4 decades-from $2.4 \%$ to $6.5 \% .{ }^{2}$ Given this slow progress, efforts have been made over the last several years to explore the antineoplastic effects of commonly prescribed medications as potential adjunctive therapies for pancreas cancer. Aspirin, metformin, statins, $\beta$-blockers and bisphosphonate have all been studied to various degrees, with the hopes of repurposing them as either preventative or therapeutic agents. Dipeptidyl-peptidase 4 (DPP-4) inhibitors have received scrutiny on the opposite end of the spectrum, as possible pro-neoplastic agents. This review will examine the hypotheses, evidence, and current state of the literature of each of these medications and their possible role in the prevention and treatment of pancreatic cancer (Table 1).

\section{ASPIRIN AND NSAIDs}

Nonsteroidal anti-inflammatory drugs (NSAIDs) including aspirin have long been considered promising chemopreventative and chemotherapeutic agents due to their antioxidant and anti-inflammatory properties. ${ }^{3}$ The major mechanism by which aspirin exerts antineoplastic effect is through inhibition of COX1/COX-2 and modulation of the NFkB or STAT3 pathway. ${ }^{4}$ Despite this biologic plausibility and clear association with a number of nonpancreatic cancers, the epidemiological evidence with regards to the prevention and treatment of pancreatic cancer is conflicting.

An initial case-control study of 1,149 cases and 5,952 controls found no statistically significant association between NSAID use and risk of pancreatic cancer regardless of duration of use (odds ratio $[\mathrm{OR}]<5$ years, $0.8,95 \%$ confidence interval [CI], 0.5 to 1.4 ; OR >5 years, $0.6,95 \%$ CI, 0.4 to 1.1$){ }^{5}$ However, this study did not separate aspirin from other NSAIDs. A followup prospective study of 28,282 postmenopausal woman did make this distinction, and found a negative association between current aspirin use, but not NSAID use, and incident pancreatic cancer (OR aspirin, $0.47,95 \% \mathrm{CI}, 0.22$ to 0.98 ; OR nonaspirin NSAID, $0.89,95 \%$ CI, 0.35 to 2.24$).{ }^{6}$ A second case-control study published several years later confirmed this result. ${ }^{7} \mathrm{Nev}$ ertheless, data in support of the chemopreventative effects of aspirin are not entirely universal. Prospective data from the Nurses' Health Study looking at aspirin alone has suggested that woman who reported more than 20 years of aspirin use have an increased risk of pancreatic cancer (1.48; 95\% CI, 1.03 to 2.43),

Correspondence to: Aimee L. Lucas

Division of Gastroenterology, Icahn School of Medicine at Mount Sinai, 1 Gustave L. Levy Place, Box 1069, New York, NY 10029, USA

Tel: +1-212-241-0101, Fax: +1-646-537-8647, E-mail: Aimee.Lucas@mssm.edu

Received on September 11, 2015. Revised on December 8, 2015. Accepted on December 9, 2015.

pISSN 1976-2283 eISSN 2005-1212 http://dx.doi.org/10.5009/gnl15451

@ This is an Open Access article distributed under the terms of the Creative Commons Attribution Non-Commercial License (http://creativecommons.org/licenses/by-nc/4.0) which permits unrestricted non-commercial use, distribution, and reproduction in any medium, provided the original work is properly cited. 
Table 1. Common Pharmaceutical Agents and Proposed Mechanisms in Pancreatic Cancer

\begin{tabular}{lll}
\hline \multicolumn{1}{c}{ Medication } & Proposed effect & \\
\hline Aspirin & Protective & Inhibit COX-1/COX-2 \\
& & Modulate NFkB or STAT3 pathway \\
Metformin & Protective & Lower insulin/IGF-1 levels \\
& & Activate AMPK which inactivates mTOR pathway \\
Statins & Protective & Prevent synthesis of mevalonic acid, which then activates small G proteins-Ras, Rho, and Rac \\
$\beta$-Blockers & Protective & Block cAMP-dependent intracellular signaling and release of EGF \\
& & Block PKA-dependent release of VEGF \\
Bisphosphonates & Protective & Interfere with RAS and Rho pathways \\
& & Inhibit tumor educated macrophages \\
DPP-4 inhibitors & Cancer promoting & Stimulate pancreatic $\beta$-cells to release insulin, resulting in increased $\alpha$ - and $\beta$-cell mass \\
\hline
\end{tabular}

IGF-1, insulin-like growth factor 1; AMPK, AMP-activated protein kinase; mTOR, mammalian target of rapamycin; EGF, epidermal growth factor; PKA, protein kinase A; VEGF, vascular endothelial growth factor; DPP-4, dipeptidyl peptidase-4.

although subgroup analyses limited this association to greater or equal to 14 tablets per week. ${ }^{8}$ In order to reconcile these results, three meta-analyses have been published. Although the first two studies show no significant association between aspirin use and incident pancreatic cancer for any exposure level, the most recent analysis of 7,252 cases and 120,000 healthy controls suggests a trend towards chemoprevention for high dose but not low dose aspirin use (pooled OR high dose, $0.88,95 \%$ CI, 0.76 to 1.01; pooled OR low dose, $0.99,95 \% \mathrm{CI}, 0.91$ to 1.07$).^{9-11}$

With regards to the chemotherapeutic potential of aspirin in pancreatic cancer, there are fewer dedicated studies. A large, pooled analysis of 25,570 patients in eight randomized trials showed a survival benefit of daily aspirin (75 mg or greater) for several common cancers including pancreatic, esophageal, lung, stomach, and colorectal. This association increased with duration of treatment. ${ }^{12}$

\section{METFORMIN, DPP-4 INHIBITORS, AND THE ANTIHYPERGLYCEMICS}

Metformin is a biguanide oral hypoglycemic used to treat type 2 diabetes mellitus. It is thought to work by decreasing hepatic glucose production and increasing insulin sensitivity. In vitro studies suggest that metformin may inhibit pancreatic cancer cells by lowering insulin and insulin-like growth factor (IGF-1) levels. These hormones may stimulate cancer cell growth via their interaction with G-protein coupled receptors. ${ }^{13,14}$ Metformin may also have direct inhibitory effects on pancreatic ductal adenocarcinoma cells by activating the AMP-activated protein kinase (AMPK), which then inactivates proteins in the mammalian target of rapamycin pathway thereby way which promoting cell proliferation. ${ }^{4,15-17}$

Case-control and cohort studies have suggested that metformin use at any time may reduce the incidence of pancreatic cancer among diabetics, whereas insulin and insulin secreta- gogues may increase this risk. ${ }^{18-21}$ A case-control study of 973 patients with pancreatic adenocarcinoma and 863 controls found a significantly lower risk of pancreatic cancer among diabetics who had taken metformin, but an increased risk among those who had taken insulin (adjusted OR metformin, 0.41, 95\% CI, 0.19 to 0.87; adjusted OR insulin, 5.04, 95\% CI, 2.38 to 10.7). ${ }^{19}$ A case-control study using the United Kingdom (U.K.) General Practice Research Database showed similar results, although the protective effect of metformin was only observed in women. ${ }^{21}$ Data from a prospective cohort of 800,000 patients from Taiwan controlling for Charlson comorbidity score, duration of metformin exposure and use of other oral antihyperglycemics also showed a protective effect of metformin use among all diabetics (adjusted hazard ratio [HR], 0.15; 95\% CI, 0.03 to 0.79). ${ }^{20}$ Despite these results however, a recent meta-analysis of 11 studies and 1,770 pancreatic cancer cases in 730,664 diabetic patients did not find an association between metformin (adjusted OR, 0.76; 95\% CI, 0.57 to 1.03), insulin, or thiazolidinediones and the risk of developing pancreatic cancer. ${ }^{22}$ Interestingly, this meta-analysis did show a 70\% increased odds of pancreatic cancer with sulfonylureas; however, significant heterogeneity was noted between studies. ${ }^{22}$

Few studies have examined the chemotherapeutic potential of metformin among patients with pancreatic cancer, and all of these studies have significant limitations. Another study of 302 patients with pancreatic cancer and diabetes, of which 117 were exposed to metformin, found the HR for death among diabetics with nonmetastatic disease for those on metformin to be 0.64 (95\% CI, 0.48 to 0.86). ${ }^{23}$ However, this study did not take into account the concurrent use of other antihyperglycemics or control for diabetic severity. Recall bias was a potential issue with this study, as detailed medical information was obtained via personal interview for $76 \%$ of patients. Similarly, data from a U.K. cohort of 516 diabetic patients with stage IV pancreatic cancer (247 of whom were exposed to metformin) found no 
difference in survival on univariate or multivariate survival analysis (HR, 1.09; 95\% CI, 0.80 to 1.47); however, this study was unable to control for race or account for insulin or other antidiabetic agents. $^{24}$

Another class of antihyperglycemic that has received considerable attention for its possible association with incident pancreatic cancer is the DPP-4 inhibitors. These medications inhibit the breakdown of the incretin hormones, glucagon-like peptide 1 (GLP-1) and gastric inhibitory peptide (GIP), which in turn lower blood glucose concentrations by stimulating pancreatic $\beta$ cells to release insulin. ${ }^{25}$ Sitagliptin was the first DPP-4 inhibitor earning Food and Drug Adminstration (FDA) approval in 2006, and several other newer drugs have since been developed. Initial enthusiasm for these medications was diffused by early studies suggesting a possible increase in pancreatitis and pancreatic cancer among diabetics receiving incretin-based therapies. Pancreatitis is a well-known risk factor for pancreatic cancer. ${ }^{26}$ Not only did preclinical studies in animal models suggest increased $\beta$-cell mass and regeneration, but autopsy studies in humans reports a fourfold increase in $\alpha$ - and $\beta$-cell mass among diabetics receiving these therapies compared to those on other therapies. ${ }^{27-29}$ An examination of the FDA's database of reported adverse events found a 6-fold increased odds of pancreatitis among those taking sitagliptin (OR, 6.74; 95\% CI, 4.61 to 10.00) compared to other diabetic medications and 10-fold increase in the odds of pancreatitis among those taking GLP-1 mimetic exenatide (OR, 10.68; 95\% CI, 7.75 to 15.10$).{ }^{30}$ Both drugs also appeared to significantly increase the risk of pancreatic cancer without increasing the risk of other cancers. Nevertheless, two subsequent large multicenter placebo controlled clinical trials (EXAMINE and SAVOR-TIMI) as well as a meta-analysis found no increased rate of acute or chronic pancreatitis with DPP-4 inhibitor therapy. ${ }^{31-33}$ Most recently, the FDA and the European Medicines Agency (EMA) published a joint statement on the association between incretin-based drugs and both pancreatitis and pancreas cancer. After exhaustive review of both preclinical and epidemiological studies, the authors conclude that current data do not support a causal relationship between DPP-4 inhibitors and either pancreatitis or pancreatic cancer. ${ }^{34}$

\section{STATINS}

Statins are 3-hydroxy-3-methyl-glutaryl-coenzyme A (HMG$\mathrm{CoA}$ ) reductase inhibitors indicated for the treatment of hypercholesterolemia and the primary and secondary prevention of coronary heart disease and its risk equivalents. ${ }^{35}$ Statins have pleiotropic effects, and their use as antineoplastic agents has been of considerable interest for several reasons. First, HMGCoA inhibition prevents the synthesis of mevalonic acid, a precursor of nonsteroidal isoprenoids, which then activate small G proteins such as Ras, Rho, and Rac. ${ }^{36}$ Ras signaling is particularly important in pancreatic tumorigenesis. ${ }^{37-39}$ Second, statins are antiangiogenic and exert proapoptotic and anti-inflammatory effects. ${ }^{40-43}$ To date, statin use has been associated with a decreased risk for a number of cancers, including colorectal, esophageal, liver, and pancreatic cancer. ${ }^{44-46}$

Similar to other commonly prescribed medications, dedicated epidemiological data on statins and the risk of pancreatic cancer are conflicting. A large case-control study of 483,733 predominantly U.S. male (98.5\%) veterans found that statin use for more than 6 months was associated with a 67\% risk reduction in incidence of pancreatic cancer (adjusted OR, 0.33; 95\% CI, 0.26 to 0.41 , whereas statin use for more than 4 years was associated with an 80\% risk reduction (adjusted OR, 0.20; 95\% CI, 0.13 to 0.29$).{ }^{47}$ However, a subsequent meta-analysis of 12 studies (three randomized placebo-controlled trials [RCTs], four cohorts, and five case-control studies) found no evidence of association between statin use and pancreatic cancer among either the RCTs (relative risk [RR], 0.99; 95\% CI, 0.44 to 2.21) or the observational studies (RR, 0.86; 95\% CI, 0.60 to 1.24 )..$^{48}$ Although no publication bias was detected, there was high heterogeneity among the observational studies and sex-specific analyses were not performed. One way to reconcile these data is the notion that the inverse association between statins and incident pancreatic cancer may be gender-specific. This position is supported by two further more recent observational studies. A smaller U.K. based case-control study found a reduced risk of pancreatic cancer only among male smokers (OR, 0.11; 95\% CI, 0.01 to 0.96), and a larger San Francisco clinic based case-control study of 536 cases and 869 controls found a similar sex-specific inverse association in males only (OR for men, 0.50, 95\% CI, 0.32 to 0.79 ; OR for women, $0.86,95 \% \mathrm{CI}, 0.52$ to 1.43$).^{49,50}$

Statins may also have a chemotherapeutic use after pancreatic cancer diagnosis. A recent population based analysis of the Surveillance, Epidemiology and End Results (SEER)-Medicare database found a $21 \%$ reduced risk of death (HR, 0.79; 95\% CI, 0.67 to 0.93 ) for statin use after diagnosis among patients with low-grade (I or II), but not high grade (III or IV) pancreatic adenocarcinoma. ${ }^{51}$ Patients who had undergone pancreatectomy, had chronic pancreatitis, and had not been treated with a statin prior to cancer diagnosis were also found to benefit from postdiagnosis statin therapy. Finally, there is emerging clinical trial investigating the role of statins as adjunctive chemotherapeutic agents in pancreatic cancer. Although a randomized, doubleblinded, placebo-controlled phase II trial of gemcitabine and simvastatin versus gemcitabine and placebo found no additional clinical benefit to statin therapy in advanced pancreatic cancer patients, no increased toxicity was noted either. ${ }^{52}$ As such, there may be a role for statin therapy in conjunction with antiepidermal growth factor receptor (anti-EGFR) agents such as erlotinib or cetuximab, or even as part of neo-adjuvant regimens in less advanced disease, although further studies are needed to investigate these possibilities. 


\section{$\beta$-BLOCKERS}

$\beta$-Blockers are a widely used class of drugs employed for the treatment of heart failure, hypertension, myocardial infarction, and arrhythmias among other disorders. $\beta$-Blockers competitively antagonize the $\beta-1$ or $\beta-2$ adrenergic receptor, which subsequently results in decreased intracellular levels of cyclic adenosine monophosphatase (cAMP). ${ }^{53} \mathrm{~B} 1$ receptors are primarily found in heart muscle, whereas B2 receptors are primarily found in the bronchus or smooth muscle of the peripheral vasculature.

A number of in vitro studies have suggested a potential role of $\beta$-blockade in the regulation of several cancers, including pancreatic, prostate, breast, colon, and ovarian. ${ }^{54-58}$ With regards to pancreas cancer, $\beta$-adrenergic agonists stimulate human ductal adenocarcinoma cell growth via adenyl cyclase, which has downstream effects on cAMP, protein kinase A (PKA), and phosphorylation of cyclic AMP response element binding protein (p-CREB). ${ }^{54,59}$ PKA also activates the EGFR pathway. Furthermore, tobacco, via the carcinogen 4-(methylnitrosamino)1-(3-pyridyl)-1-butanone (NNK), may also stimulate pancreatic cell growth via this same $\beta$-adrenergic mechanism. ${ }^{54,60}$ In an animal model of hamsters with ethanol-induced pancreatitis, the nonselective $\beta$-blocker propranalol showed strong antineoplastic effect, inhibiting upregulation of the $\alpha 7$ nicotinic acetylcholine receptor $(\alpha 7 \mathrm{nAChR})$ as well the extracellular signal regulated protein kinases (ERK1/2), p-CREB, EGF, and vascular endothelial growth factor. ${ }^{54}$

No dedicated epidemiological studies have examined the effects of $\beta$-blockade on pancreatic cancer incidence or survival. However, a retrospective study of the U.K. primary care Doctors' Independent Network database examining patients with several cancers found an effect opposite to that suggested by in vitro studies. Among patients with pancreatic and prostate cancer, those on $\beta$-blockers had worse overall survival than those on other antihypertensive medications (HR pancreatic cancer, 1.88; 95\% CI, 1.09 to 3.25). ${ }^{61}$ Nevertheless, a subsequent study of the parallel Clinical Practice Research Datalink found no such effect of $\beta$-blocker use among patients with pancreatic cancer (HR, $1.04 ; 95 \%$ CI, 0.92 to 1.08$){ }^{62}$

\section{BISPHOSPHONATES}

Bisphosphonates inhibit osteoclast-mediated bone resorption, and are indicated for the prevention and treatment of osteoporosis. The prescribing of bisphosphonates has increased substantially in Western countries over the last 2 decades, especially in older women. ${ }^{63,64}$ A number of preclinical studies suggest a potential role of bisphosphonates as antineoplastic agents against a wide variety of cancers, and several clinical trials have explored the role of these medications as adjuvant therapy in breast cancer. ${ }^{65-67}$ In pancreatic cancer cells in particular, bisphosphonates have been shown to induce antiproliferative, antiapoptic, and antimetastatic effects via interference with the RAS and Rho pathways and the inhibition of tumor educated macrophages. $^{68-71}$

There is minimal epidemiological data on the association between bisphosphonate use and incident pancreatic cancer. A case-control study of 41,826 patients from the U.K. general practice database found a reduced incidence of all cancer after any bisphosphonate use (HR, 0.87; 95\% CI, 0.82 to 0.92), however no statistically significant reduction in risk of pancreatic cancer (HR, 0.91; 95\% CI, 0.53 to 1.35$).{ }^{72}$ A large nested-case control study of two other large U.K. databases compromising 180,000 cases (matched with 1:5 with controls) however, did find an inverse association between bisphosphonate use and incident pancreatic cancer (adjusted OR, 0.79; 95\% CI, 0.68 to 0.93) when both cohorts were combined. ${ }^{73}$

To date, no clinical trials or epidemiological studies have explored the chemotherapeutic role of bisphosphonates in patients with pancreatic cancer. However, early data suggest that the combination of zoledronic acid and gemcitabine may inhibit proliferation and invasion of pancreatic cells in vitro, and tumor growth and the development of liver metastases in athymic mice with implanted pancreatic cancer cell lines. ${ }^{74}$

\section{CONCLUSIONS}

Survival from pancreatic cancer remains exceedingly poor. Conventional treatment has resulted in only marginal improvements in survival over the last several decades. As such, screening and prevention have taken a prominent role in the approach to the management of this disease. Specifically, the medical community has focused considerable attention on the re-purposing of common pharmaceutical agents as possible chemopreventative and chemotherapeutic options. Aspirin, metformin, statins, $\beta$-blockers and bisphosphonates all have biological plausible mechanisms to inhibit pancreatic neoplasia, whereas DDP-4 inhibitors may promote it. Unfortunately, epidemiological evidence in support of these hypotheses remains moderate at best. Aspirin, but not NSAIDs, may have slightly reduced risk of incidence pancreatic cancer, but not to a clinically relevant degree. Metformin may reduce the risk of pancreatic cancer in diabetics, but data from observational studies and meta-analyses are conflicting. With regards to survival, metformin does not appear to benefit diabetics with advanced disease; however, its role in locoregional disease and in nondiabetics as a possible chemopreventative agent remains unknown. Statins appear to exert a weak, but not insignificant male-specific protective effect on incident pancreatic cancer for unclear reasons. The role of statins as adjunctive chemotherapeutic agents is currently being studied. $\beta$-Blockers have strong in vitro and animal data to suggest a protective effect, but the few population studies performed have not supported this hypothesis. With regards 
to bisphosphonates, large population studies have shown only minimal associations with incident pancreatic cancer, and the potential chemotherapeutic role of this class, though biologically promising, remains untested at present. Finally, although early data suggested an increased risk of pancreatitis and pancreatic cancer with incretin-based therapies (DPP-4 inhibitors and GLP agonists), more recent clinical trials, meta-analytical data, and a combined FDA/EMA statement argue against any causal relationship.

\section{CONFLICTS OF INTEREST}

No potential conflict of interest relevant to this article are reported.

\section{REFERENCES}

1. Siegel R, Ma J, Zou Z, Jemal A. Cancer statistics, 2014. CA Cancer J Clin 2014;64:9-29.

2. Howlader N, Noone AM, Krapcho M, et al. SEER cancer statistics review (CSR) 1975-2010. Bethesda: National Cancer Institute, 2013.

3. Ulrich CM, Bigler J, Potter JD. Non-steroidal anti-inflammatory drugs for cancer prevention: promise, perils and pharmacogenetics. Nat Rev Cancer 2006;6:130-140.

4. Yue W, Yang CS, DiPaola RS, Tan XL. Repurposing of metformin and aspirin by targeting AMPK-mTOR and inflammation for pancreatic cancer prevention and treatment. Cancer Prev Res (Phila) 2014;7:388-397.

5. Coogan PF, Rosenberg L, Palmer JR, et al. Nonsteroidal anti-inflammatory drugs and risk of digestive cancers at sites other than the large bowel. Cancer Epidemiol Biomarkers Prev 2000;9:119123.

6. Anderson KE, Johnson TW, Lazovich D, Folsom AR. Association between nonsteroidal anti-inflammatory drug use and the incidence of pancreatic cancer. J Natl Cancer Inst 2002;94:1168-1171.

7. Tan XL, Reid Lombardo KM, Bamlet WR, et al. Aspirin, nonsteroidal anti-inflammatory drugs, acetaminophen, and pancreatic cancer risk: a clinic-based case-control study. Cancer Prev Res (Phila) 2011;4:1835-1841.

8. Schernhammer ES, Kang JH, Chan AT, et al. A prospective study of aspirin use and the risk of pancreatic cancer in women. J Natl Cancer Inst 2004;96:22-28.

9. Larsson SC, Giovannucci E, Bergkvist L, Wolk A. Aspirin and nonsteroidal anti-inflammatory drug use and risk of pancreatic cancer: a meta-analysis. Cancer Epidemiol Biomarkers Prev 2006;15:2561-2564.

10. Capurso G, Schünemann HJ, Terrenato I, et al. Meta-analysis: the use of non-steroidal anti-inflammatory drugs and pancreatic cancer risk for different exposure categories. Aliment Pharmacol Ther 2007;26:1089-1099.

11. Cui XJ, He Q, Zhang JM, Fan HJ, Wen ZF, Qin YR. High-dose aspirin consumption contributes to decreased risk for pancreatic cancer in a systematic review and meta-analysis. Pancreas 2014;43:135-140.

12. Rothwell PM, Fowkes FG, Belch JF, Ogawa H, Warlow CP, Meade TW. Effect of daily aspirin on long-term risk of death due to cancer: analysis of individual patient data from randomised trials. Lancet 2011;377:31-41.

13. Rozengurt E. Mitogenic signaling pathways induced by G proteincoupled receptors. J Cell Physiol 2007;213:589-602.

14. Nakamura K, Sasajima J, Mizukami Y, et al. Hedgehog promotes neovascularization in pancreatic cancers by regulating Ang-1 and IGF-1 expression in bone-marrow derived pro-angiogenic cells. PLoS One 2010;5:e8824.

15. Inoki K, Ouyang H, Zhu T, et al. TSC2 integrates Wnt and energy signals via a coordinated phosphorylation by AMPK and GSK3 to regulate cell growth. Cell 2006;126:955-968.

16. Fryer LG, Parbu-Patel A, Carling D. The anti-diabetic drugs rosiglitazone and metformin stimulate AMP-activated protein kinase through distinct signaling pathways. J Biol Chem 2002;277:25226-25232.

17. Kisfalvi K, Eibl G, Sinnett-Smith J, Rozengurt E. Metformin disrupts crosstalk between $G$ protein-coupled receptor and insulin receptor signaling systems and inhibits pancreatic cancer growth. Cancer Res 2009;69:6539-6545.

18. Currie CJ, Poole CD, Gale EA. The influence of glucose-lowering therapies on cancer risk in type 2 diabetes. Diabetologia 2009;52:1766-1777.

19. Li D, Yeung SC, Hassan MM, Konopleva M, Abbruzzese JL. Antidiabetic therapies affect risk of pancreatic cancer. Gastroenterology 2009;137:482-488.

20. Lee MS, Hsu CC, Wahlqvist ML, Tsai HN, Chang YH, Huang YC. Type 2 diabetes increases and metformin reduces total, colorectal, liver and pancreatic cancer incidences in Taiwanese: a representative population prospective cohort study of 800,000 individuals. BMC Cancer 2011;11:20.

21. Bodmer M, Becker C, Meier C, Jick SS, Meier CR. Use of antidiabetic agents and the risk of pancreatic cancer: a case-control analysis. Am J Gastroenterol 2012;107:620-626.

22. Singh S, Singh PP, Singh AG, Murad MH, McWilliams RR, Chari ST. Anti-diabetic medications and risk of pancreatic cancer in patients with diabetes mellitus: a systematic review and metaanalysis. Am J Gastroenterol 2013;108:510-519.

23. Sadeghi N, Abbruzzese JL, Yeung SC, Hassan M, Li D. Metformin use is associated with better survival of diabetic patients with pancreatic cancer. Clin Cancer Res 2012;18:2905-2912.

24. Hwang AL, Haynes K, Hwang WT, Yang YX. Metformin and survival in pancreatic cancer: a retrospective cohort study. Pancreas 2013;42:1054-1059.

25. Tella SH, Rendell MS. DPP-4 inhibitors: focus on safety. Expert Opin Drug Saf 2015;14:127-140.

26. Rebours V, Boutron-Ruault MC, Schnee M, et al. The natural history of hereditary pancreatitis: a national series. Gut 2009;58:97- 
103.

27. Butler AE, Campbell-Thompson M, Gurlo T, Dawson DW, Atkinson M, Butler PC. Response to comments on: Butler et al. Marked expansion of exocrine and endocrine pancreas with incretin therapy in humans with increased exocrine pancreas dysplasia and the potential for glucagon-producing neuroendocrine tumors. Diabetes 2013;62:2595-2604. Diabetes 2013;62:e19-e22.

28. Lee YS, Jun HS. Anti-diabetic actions of glucagon-like peptide-1 on pancreatic beta-cells. Metabolism 2014;63:9-19.

29. Rondas D, D’Hertog W, Overbergh L, Mathieu C. Glucagon-like peptide-1: modulator of beta-cell dysfunction and death. Diabetes Obes Metab 2013;15 Suppl 3:185-192.

30. Elashoff M, Matveyenko AV, Gier B, Elashoff R, Butler PC. Pancreatitis, pancreatic, and thyroid cancer with glucagon-like peptide1-based therapies. Gastroenterology 2011;141:150-156.

31. White WB, Cannon CP, Heller SR, et al. Alogliptin after acute coronary syndrome in patients with type 2 diabetes. N Engl J Med 2013;369:1327-1335.

32. Scirica BM, Bhatt DL, Braunwald E, et al. Saxagliptin and cardiovascular outcomes in patients with type 2 diabetes mellitus. $\mathrm{N}$ Engl J Med 2013;369:1317-1326.

33. Monami M, Dicembrini I, Mannucci E. Dipeptidyl peptidase-4 inhibitors and pancreatitis risk: a meta-analysis of randomized clinical trials. Diabetes Obes Metab 2014;16:48-56.

34. Egan AG, Blind E, Dunder K, et al. Pancreatic safety of incretin-based drugs: FDA and EMA assessment. N Engl J Med 2014;370:794-797.

35. Stone NJ, Robinson JG, Lichtenstein AH, et al. 2013 ACC/AHA guideline on the treatment of blood cholesterol to reduce atherosclerotic cardiovascular risk in adults: a report of the American College of Cardiology/American Heart Association Task Force on Practice Guidelines. J Am Coll Cardiol 2014;63(25 Pt B):2889-2934.

36. Vallianou NG, Kostantinou A, Kougias M, Kazazis C. Statins and cancer. Anticancer Agents Med Chem 2014;14:706-712.

37. di Magliano MP, Logsdon CD. Roles for KRAS in pancreatic tumor development and progression. Gastroenterology 2013;144:12201229.

38. Almoguera C, Shibata D, Forrester K, Martin J, Arnheim N, Perucho M. Most human carcinomas of the exocrine pancreas contain mutant c-K-ras genes. Cell 1988;53:549-554.

39. Rall CJ, Yan YX, Graeme-Cook F, et al. Ki-ras and p53 mutations in pancreatic ductal adenocarcinoma. Pancreas 1996;12:10-17.

40. Park HJ, Kong D, Iruela-Arispe L, Begley U, Tang D, Galper JB. 3-Hydroxy-3-methylglutaryl coenzyme A reductase inhibitors interfere with angiogenesis by inhibiting the geranylgeranylation of RhoA. Circ Res 2002;91:143-150.

41. Weis M, Heeschen C, Glassford AJ, Cooke JP. Statins have biphasic effects on angiogenesis. Circulation 2002;105:739-745.

42. Bocci G, Fioravanti A, Orlandi P, et al. Fluvastatin synergistically enhances the antiproliferative effect of gemcitabine in human pancreatic cancer MIAPaCa-2 cells. Br J Cancer 2005;93:319-330. 43. Dulak J, Józkowicz A. Anti-angiogenic and anti-inflammatory effects of statins: relevance to anti-cancer therapy. Curr Cancer Drug Targets 2005;5:579-594.

44. Poynter JN, Gruber SB, Higgins PD, et al. Statins and the risk of colorectal cancer. N Engl J Med 2005;352:2184-2192.

45. Singh S, Singh AG, Singh PP, Murad MH, Iyer PG. Statins are associated with reduced risk of esophageal cancer, particularly in patients with Barrett's esophagus: a systematic review and metaanalysis. Clin Gastroenterol Hepatol 2013;11:620-629.

46. Shi M, Zheng H, Nie B, Gong W, Cui X. Statin use and risk of liver cancer: an update meta-analysis. BMJ Open 2014;4:e005399.

47. Khurana V, Sheth A, Caldito G, Barkin JS. Statins reduce the risk of pancreatic cancer in humans: a case-control study of half a million veterans. Pancreas 2007;34:260-265.

48. Bonovas S, Filioussi K, Sitaras NM. Statins are not associated with a reduced risk of pancreatic cancer at the population level, when taken at low doses for managing hypercholesterolemia: evidence from a meta-analysis of 12 studies. Am J Gastroenterol 2008;103:2646-2651

49. Carey FJ, Little MW, Pugh TF, et al. The differential effects of statins on the risk of developing pancreatic cancer: a casecontrol study in two centres in the United Kingdom. Dig Dis Sci 2013;58:3308-3312.

50. Walker EJ, Ko AH, Holly EA, Bracci PM. Statin use and risk of pancreatic cancer: results from a large, clinic-based case-control study. Cancer 2015;121:1287-1294.

51. Jeon CY, Pandol SJ, Wu B, et al. The association of statin use after cancer diagnosis with survival in pancreatic cancer patients: a SEER-medicare analysis. PLoS One 2015;10:e0121783.

52. Hong JY, Nam EM, Lee J, et al. Randomized double-blinded, placebo-controlled phase II trial of simvastatin and gemcitabine in advanced pancreatic cancer patients. Cancer Chemother Pharmacol 2014;73:125-130.

53. Akbar S, Alsharidah MS. Are beta blockers new potential anticancer agents? Asian Pac J Cancer Prev 2014;15:9567-9574.

54. Al-Wadei HA, Al-Wadei MH, Schuller HM. Prevention of pancreatic cancer by the beta-blocker propranolol. Anticancer Drugs 2009;20:477-482.

55. Palm D, Lang K, Niggemann B, et al. The norepinephrine-driven metastasis development of PC-3 human prostate cancer cells in $\mathrm{BALB} / \mathrm{c}$ nude mice is inhibited by beta-blockers. Int $\mathrm{J}$ Cancer 2006;118:2744-2749.

56. Drell TL IV, Joseph J, Lang K, Niggemann B, Zaenker KS, Entschladen F. Effects of neurotransmitters on the chemokinesis and chemotaxis of MDA-MB-468 human breast carcinoma cells. Breast Cancer Res Treat 2003;80:63-70.

57. Masur K, Niggemann B, Zanker KS, Entschladen F. Norepinephrine-induced migration of SW 480 colon carcinoma cells is inhibited by beta-blockers. Cancer Res 2001;61:2866-2869.

58. Sood AK, Bhatty R, Kamat AA, et al. Stress hormone-mediated invasion of ovarian cancer cells. Clin Cancer Res 2006;12:369-375.

59. Weddle DL, Tithoff P, Williams M, Schuller HM. Beta-adrenergic growth regulation of human cancer cell lines derived from pan- 
creatic ductal carcinomas. Carcinogenesis 2001;22:473-479.

60. Askari MD, Tsao MS, Schuller HM. The tobacco-specific carcinogen, 4-(methylnitrosamino)-1-(3-pyridyl)-1-butanone stimulates proliferation of immortalized human pancreatic duct epithelia through beta-adrenergic transactivation of EGF receptors. J Cancer Res Clin Oncol 2005;131:639-648.

61. Shah SM, Carey IM, Owen CG, Harris T, Dewilde S, Cook DG. Does beta-adrenoceptor blocker therapy improve cancer survival? Findings from a population-based retrospective cohort study. Br J Clin Pharmacol 2011;72:157-161.

62. Springate DA, Ashcroft DM, Kontopantelis E, Doran T, Ryan R, Reeves D. Can analyses of electronic patient records be independently and externally validated? Study 2: the effect of betaadrenoceptor blocker therapy on cancer survival: a retrospective cohort study. BMJ Open 2015;5:e007299.

63. Udell JA, Fischer MA, Brookhart MA, Solomon DH, Choudhry NK. Effect of the women's health initiative on osteoporosis therapy and expenditure in Medicaid. J Bone Miner Res 2006;21:765-71.

64. Watson J, Wise L, Green J. Prescribing of hormone therapy for menopause, tibolone, and bisphosphonates in women in the UK between 1991 and 2005. Eur J Clin Pharmacol 2007;63:843-849.

65. Diel IJ, Solomayer EF, Costa SD, et al. Reduction in new metastases in breast cancer with adjuvant clodronate treatment. $\mathrm{N}$ Engl $\mathrm{J}$ Med 1998;339:357-363.

66. Powles T, Paterson A, McCloskey E, et al. Reduction in bone relapse and improved survival with oral clodronate for adjuvant treatment of operable breast cancer [ISRCTN83688026]. Breast Cancer Res 2006;8:R13.
67. Gnant M, Mlineritsch B, Luschin-Ebengreuth G, et al. Adjuvant endocrine therapy plus zoledronic acid in premenopausal women with early-stage breast cancer: 5-year follow-up of the ABCSG-12 bone-mineral density substudy. Lancet Oncol 2008;9:840-849.

68. Tassone P, Tagliaferri P, Viscomi C, et al. Zoledronic acid induces antiproliferative and apoptotic effects in human pancreatic cancer cells in vitro. Br J Cancer 2003;88:1971-1978.

69. Takiguchi S, Nishino Y, Inoue K, et al. The bisphosphonate incadronate inhibits intraperitoneal dissemination in an in vivo pancreatic cancer model. Oncol Rep 2012;28:111-116.

70. Hiroshima Y, Maawy A, Hassanein MK, et al. The tumor-educatedmacrophage increase of malignancy of human pancreatic cancer is prevented by zoledronic acid. PLoS One 2014;9:e103382.

71. Märten A, Lilienfeld-Toal Mv, Büchler MW, Schmidt J. Zoledronic acid has direct antiproliferative and antimetastatic effect on pancreatic carcinoma cells and acts as an antigen for delta2 gamma/ delta T cells. J Immunother 2007;30:370-377.

72. Cardwell CR, Abnet CC, Veal P, Hughes CM, Cantwell MM, Murray LJ. Exposure to oral bisphosphonates and risk of cancer. Int $\mathrm{J}$ Cancer 2012;131:E717-E725.

73. Vinogradova Y, Coupland C, Hippisley-Cox J. Exposure to bisphosphonates and risk of common non-gastrointestinal cancers: series of nested case-control studies using two primary-care databases. Br J Cancer 2013;109:795-806.

74. Zhao M, Tominaga Y, Ohuchida K, et al. Significance of combination therapy of zoledronic acid and gemcitabine on pancreatic cancer. Cancer Sci 2012;103:58-66. 